\title{
NIR-Emitting Alloyed CdTeSe QDs and Organic Dye Assemblies: A Nontoxic, Stable, and Efficient FRET System
}

\author{
Doris E. Ramírez-Herrera ${ }^{1}$, Eustolia Rodríguez-Velázquez ${ }^{2,3}$, Manuel Alatorre-Meda ${ }^{3,4}$, \\ Francisco Paraguay-Delgado ${ }^{5}$, Antonio Tirado-Guízar ${ }^{1}$, Pablo Taboada ${ }^{6}$ \\ and Georgina Pina-Luis ${ }^{1, *}$
}

1 Centro de Graduados e Investigación, Instituto Tecnológico de Tijuana, A.P. 1166, 22500 Tijuana, BC, Mexico; doris_e_777@hotmail.com (D.E.R.-H.); antonio.tirado@tectijuana.edu.mx (A.T.-G.)

2 Facultad de Odontología, Universidad Autónoma de Baja California, Calzada Universidad 14418, Parque Industrial Internacional, 22390 Tijuana, BC, Mexico; eustolia.rodriguez@uabc.edu.mx

3 Instituto de Ortopedia y Banco de Tejidos Musculoesqueléticos, Universidad de Santiago de Compostela, Campus Sur S/N, E-15782 Santiago de Compostela, Spain

4 CONACyT-Instituto Tecnológico de Tijuana, Centro de Graduados e Investigación en Química, Blvd. Alberto Limón Padilla S/N, 22510 Tijuana, BC, Mexico; malatorreme@conacyt.mx

5 Centro de Investigación en Materiales Avanzados S. C., Departamento de Física de Materiales, Av. Miguel de Cervantes 120, Complejo Industrial Chihuahua, CP 31109 Chihuahua, Chih., Mexico; francisco.paraguay@cimav.edu.mx

6 Grupo de Física de Coloides y Polímeros, Departamento de Física de Materia Condensada, Facultad de Física, Universidad de Santiago de Compostela, Campus Sur S/N, E-15782 Santiago de Compostela, Spain; pablo.taboada@usc.es

* Correspondence: gpinaluis@tectijuana.mx; Tel.: +52-664-6233-772; Fax: +52-664-6234-341

Received: 28 February 2018; Accepted: 30 March 2018; Published: 11 April 2018

\begin{abstract}
In the present work, we synthesize Near Infrared (NIR)-emitting alloyed mercaptopropionic acid (MPA)-capped CdTeSe quantum dots (QDs) in a single-step one-hour process, without the use of an inert atmosphere or any pyrophoric ligands. The quantum dots are water soluble, non-toxic, and highly photostable and have high quantum yields (QYs) up to 84\%. The alloyed MPA-capped CdTeSe QDs exhibit a red-shifted emission, whose color can be tuned between visible and NIR regions $(608-750 \mathrm{~nm})$ by controlling the Te:Se molar ratio in the precursor mixtures and/or changing the time reaction. The MPA-capped QDs were characterized by UV-visible absorption spectroscopy, fluorescence spectroscopy, transmission electron microscopy (TEM), energy dispersive X-ray spectroscopy (EDS), and zeta potential measurements. Photostability studies were performed by irradiating the QDs with a high-power xenon lamp. The ternary MPA-CdTeSe QDs showed greater photostability than the corresponding binary MPA-CdTe QDs. We report the Förster resonance energy transfer (FRET) from the MPA-capped CdTeSe QDs as energy donors and Cyanine5 NHS-ester (Cy5) dye as an energy acceptor with efficiency $(E)$ up to 95\%. The distance between the QDs and dye $(r)$, the Förster distance $\left(R_{0}\right)$, and the binding constant $(K)$ are reported. Additionally, cytocompatibility and cell internalization experiments conducted on human cancer cells (HeLa) cells revealed that alloyed MPA-capped CdTeSe QDs are more cytocompatible than MPA-capped CdTe QDs and are capable of ordering homogeneously all over the cytoplasm, which allows their use as potential safe, green donors for biological FRET applications.
\end{abstract}

Keywords: energy transfer; alloyed quantum dots; quantum dots-dye assemblies; cytocompatibility 


\section{Introduction}

Quantum dots (QDs) are considered an excellent alternative to organic fluorophores due to their unique optical properties, such as broad excitation spectra, high photostability, size-tunable emission, narrow and symmetric emission spectra, and high quantum yields (QYs) [1]. These characteristics demonstrate them as attractive materials for biomedical and technological applications, such as clinical diagnosis and labeling [2-4], as well as for solar energy conversion in photovoltaic devices [5,6].

Regarding their tentative use in the biomedical field, QDs are well-known to respond in the red to near-infrared (NIR) window (600-900 nm), which is highly advantageous for in vitro and in vivo imaging and detection [7]. NIR light easily penetrates animal tissue due to the minimum absorbance of NIR photons [8]. Ternary alloyed QDs have recently emerged as a new class of NIR emitters attracting considerable attention, due to the capability of tuning their optical emission without changing the particle size. Examples of ternary NIR QDs are $\mathrm{CuInS}_{2}, \mathrm{AgInSe} 2$, and CdTeSe [9]. Several groups have investigated the synthesis of NIR-emitting CdTeSe QDs [10-14]. Most of these studies are based on core-shell QD synthesis, since this type of QD shows greater photostability and QYs, but their synthesis is complicated and time-consuming. Because of this, a systematic study to obtain only core-doped QDs with high QYs and photostability is required.

Synthetic methods involving organic solvents such as trioctylphosphine (TOP) and trioctylphosphine oxide (TOPO) [15] have produced QDs soluble in non-polar solvents exclusively. This condition limits their application in the biomedical arena. Surface modification represents an alternative synthetic strategy to obtain water-soluble and less toxic QDs [16,17]; however, it is known that ligand surface modification fails to maintain the high QYs of QDs. An important strategy is the direct synthesis of water-soluble QDs, in a single step, eliminating the superficial exchange of ligands that leads to significant decreases of the QY. This strategy has been used in the hydrothermal synthesis of water-soluble binary [18,19] and ternary [20] QDs with good results, using mercapto acids as stabilizers. In particular, Ma et al. [18,19] prepared high-quality water-soluble CdTe QDs and investigated the influence of different mercapto acids on the growth rate, size distribution, fluorescence, and stability of the QDs. Liu et al. [20] presented ternary mercaptopropionic acid (MPA)-capped CuInS 2 QDs with QYs of 3.3\% and studied the influence of various experimental variables, including the precursor concentrations, reaction time, reaction temperature, $\mathrm{pH}$ value, and capping ligand used on the luminescent properties of the obtained QDs. These ternary QDs were used to label liver cancer cells.

Another important aspect to consider is the possible toxicity of this type of QD. Some QDs have been found to be cytotoxic only after oxidative and/or photolytic degradation of their core coatings, demonstrating that core cover greatly improves the biocompatibility of QDs with no observed cytotoxicity even at very high concentration and long-time exposure in cells [21]. Several strategies have been developed to optimize QD stability and biocompatibility. Ternary alloyed QDs represent a promising alternative due to their excellent optoelectronic properties such as: higher QY, tunable emission wavelength controlling Te:Se stoichiometry without significant change in the particle size, wide absorption range, higher chemical and structural stabilities due to decreased interdiffusion, and hardened lattice structure [22]. These advantages may contribute to a decrease in QD cytotoxicity. The stability and cytotoxicity of alloyed QDs have been less studied than binary QDs. Most cytotoxicity approaches and cell internalization studies have been performed on core-shell QDs [23-26], while the potential cytotoxicity of ternary alloyed QDs remains to be investigated thoroughly $[27,28]$. Therefore, we begin this work by carrying out a systematic study to obtain water-soluble shell-less alloyed MPA-coated QDs, in a single step, with high QYs and photostability. An important issue is to determine whether the third element in ternary QDs influences their toxicological properties, as well as the influence of surface coating, hydrodynamic diameter, and the particle surface charge.

On the other hand, Förster resonance energy transfer (FRET) mechanism has been widely used in different applications and constitutes the base of a new generation of luminescent sensors. QDs have shown great potential as fluorophores in FRET-based sensing applications, and they have been widely adopted as either energy donors or acceptors [29,30]. Although there are numerous applications of 
QD-based FRET probes, as far as we know, no studies have been reported demonstrating the use of ternary MPA-capped CdTeSe QDs in FRET systems.

In this work, we report a systematic study on the synthesis of high QY NIR-emitting MPA-capped CdTeSe QDs without the use of an inert atmosphere and pyrophoric ligands in a single step and in less than an hour. Photostability studies were performed to evaluate the change of fluorescence properties. We used a model based on MPA-capped CdTeSe QDs as energy donors and Cyanine5 NHS-ester (Cy5) dye as an energy acceptor in FRET assays. At pH 7, both species are linked by electrostatic interactions. The effect of QD size on the FRET process between nanoparticles and dye was studied. Finally, cytotoxicity and cell internalization of binary CdTe and ternary CdTeSe QD formulations were tested upon incubation with human cancer cells (HeLa).

\section{Materials and Methods}

\subsection{Materials and Characterization}

All materials used in the current work were reagents of analytical grade. Cadmium chloride hemi(pentahydrate) $\left(\mathrm{CdCl}_{2}+2.5 \mathrm{H}_{2} \mathrm{O}, 81 \%\right)$, potassium tellurite $\left(\mathrm{K}_{2} \mathrm{TeO}_{3}, 90+\%\right)$, 3-mercaptopropionic acid (MPA, $99+\%)$, sodium borohydride $\left(\mathrm{NaBH}_{4}, 99.99+\%\right)$, sodium hydroxide $(97 \%)$, Cyanine 5 NHS-ester (Cy5), and fluorescein were purchased from Sigma-Aldrich Chemicals Co. (Toluca, Edo. de Mexico, Mexico). Other reagents (analytical grade) and solvents (spectroscopic grade) were purchased from Sigma-Aldrich Chemicals Co. (Toluca, Edo. de Mexico, Mexico).

The excitation and emission spectra were recorded on a Horiba NanoLog fluorescence spectrophotometer (Kyoto, Japan) using a Xe lamp as the excitation source. QY was determined using fluorescein with a QY of $79 \%$ in $\mathrm{NaOH} 0.1 \mathrm{M}$ as a reference. The absorption spectra were measured using a Varian Cary-100 UV-visible spectrophotometer (Santa Clara, CA, USA). A Jeol JEM2200FS transmission electron microscope (TEM) (Akishima, Japan) was used to examine the appearance and size of nanoparticles. The microscope has spherical aberration correction in a scanning transmission electron microscopy (STEM mode, Akishima, Japan) working at an accelerating voltage of $200 \mathrm{KeV}$. The elemental composition was determined by energy dispersive spectroscopy (EDS) Oxford (Abingdon, UK), in which the qualitative elemental analysis was made in the STEM mode. Zeta potential was obtained on a Horiba Scientific SZ-100 (Kyoto, Japan) nanoparticle analyzer. All pH measurements were made with a Thermo Scientific $\mathrm{pH}$ meter (Waltham, MA, USA).

\subsection{Synthesis of MPA-Capped CdTeSe QDs}

Water-soluble and highly fluorescent MPA-capped CdTe and CdTeSe nanocrystals were synthesized according to the previously described method with some modifications [31]. Briefly, MPA $(0.4 \mathrm{mmol})$ and $\mathrm{CdCl}_{2}+2.5 \mathrm{H}_{2} \mathrm{O}(0.4 \mathrm{mmol})$ were dissolved in $100 \mathrm{~mL}$ deionized water in a three-necked flask. Under magnetic stirring, the $\mathrm{pH}$ of the mixture was adjusted to 10 by using the drop-wise addition of $\mathrm{NaOH}$ solution $(1 \mathrm{M})$. After 5 min of vigorous stirring, $100 \mathrm{~mL}$ of a solution prepared from $\mathrm{K}_{2} \mathrm{TeO}_{3}$ and elemental Se of different molar ratios of Te:Se (1:0, 0.75:0.25, and 0.50:0.50) and $\mathrm{NaBH}_{4}(4.2 \mathrm{mmol})$ were added into the mixture. After another $5 \mathrm{~min}$ of stirring, the flask was attached to a condenser and refluxed at $100{ }^{\circ} \mathrm{C}$. Aliquots were taken from the solution at different refluxing times $(30,45$, and $60 \mathrm{~min})$ to obtain QDs of different sizes. The as-prepared MPA-capped QD solutions were concentrated and purified by ethanol precipitation, collected via centrifugation at $3300 \mathrm{rpm}$, and re-dispersed in water. Concentration of QDs was estimated spectrophotometrically [32]. The QYs of the synthetized MPA-capped QDs were determined through Equation (1):

$$
\phi_{x}=\phi_{S T}\left(\frac{\operatorname{Grad}_{x}}{\operatorname{Grad}_{S T}}\right)\left(\frac{n_{x}^{2}}{n_{S T}^{2}}\right)
$$

where the subscripts $S T$ and $x$ denote standard and test, respectively, $\phi$ is the fluorescence quantum yield, Grad the gradient from the plot of integrated fluorescence intensity versus absorbance, and $n$ the 
refractive index of the solvent. The standard sample was fluorescein with a QY of $79 \%$ in $\mathrm{NaOH}$ solution $(0.1 \mathrm{M})$.

\subsection{MPA-Capped CdTeSe QDs and Dye Conjugates}

Donor MPA-capped CdTeSe QDs were chosen according to the spectral overlap of their emission spectra with the absorption spectra of the acceptor $\mathrm{Cy} 5$ dye. The $\mathrm{pH}$ of the solution was maintained at 7 using 2-(N-morpholino)ethanesulfonic acid (MES)-buffer (10 mM). At this $\mathrm{pH}$ value, the donor and acceptor have negative and positive surface charge, respectively.

\subsection{Titration of MPA-CdTeSe QDs with Cy5 Dye}

Cyanine5 NHS-ester (Cy5) dye stock solutions were prepared in water $(100 \mu \mathrm{M}) .3 \mathrm{~mL}$ of QD solution $(0.5 \mu \mathrm{M})$ were titrated by successive addition of dye stock solution under stirring and their resulting emission spectra were recorded.

\subsection{CCK-8 Assay}

The cytocompatibility of MPA-capped CdTe and CdTeSe QDs was determined through the CCK- 8 assay. The CCK- 8 is a sensitive colorimetric test that allows a rapid quantification of metabolically active cells in a culture, which consists of the reduction of a WST-8 tetrazolium salt into a water-soluble formazan product (light brown in color). The amount of the colored formazan product is proportional to the number of viable cells [33]. Briefly, HeLa cells with an optical confluence of $80-90 \%$ were seeded into 96-well plates $\left(100 \mu \mathrm{L}, 1.5 \times 10^{4}\right.$ cells/well) and grown for $11 \mathrm{~h}$ at standard culture conditions $\left(5 \% \mathrm{CO}_{2}\right.$ at $\left.37^{\circ} \mathrm{C}\right)$ in Dulbecco's Modified Eagle Medium (DMEM) supplemented with $10 \%$ fetal calf serum (FCS), $2 \mathrm{mM}$ L-glutamine, $1 \%$ penicillin/streptomycin, $1 \mathrm{mM}$ sodium pyruvate, and $0.1 \mathrm{mM}$ MEM non-essential amino acids (NEAA). Afterwards, the MPA-capped QDs, dissolved in $10 \mathrm{mM}$ phosphate buffered saline (PBS), were pipetted into the cell-containing wells $(100 \mu \mathrm{L}$, at varying concentrations) and incubated for $8 \mathrm{~h}$. The medium containing the MPA-capped QDs $(200 \mu \mathrm{L})$ was exchanged with fresh medium $(100 \mu \mathrm{L})$, and the cells were further incubated to complete $24 \mathrm{~h}$. Then, the culture medium was discarded, and the cells were added to a fresh culture medium $(100 \mu \mathrm{L})$ containing the CCK-8 reagent $(10 \mu \mathrm{L})$, followed by gentle shaking for $1 \mathrm{~min}$ and incubation for up to 3 $h$ at standard culture conditions. The optical density (OD) of the formazan was measured afterward at $450 \mathrm{~nm}$ using an ELISA microplate reader (BIO-RAD model 680, Hercules, CA, USA). The metabolic activity of the cells after exposure to the MPA-capped QDs, represented as the percentage of cell viability, was calculated by normalizing the formazan OD reading from the cells exposed to the MPA-capped QDs regarding control, non-exposed cells (100\% viability). The results are the average of six independent experiments.

\subsection{Confocal Microscopy}

The cellular internalization of the MPA-capped CdTeSe QDs was recorded by using a LEICA TCS-SP5 confocal microscope (LEICA Microsystems Heidelberg GmbH, Wetzlar, Hesse, Germany) equipped with a blue diode $(\lambda=405 \mathrm{~nm})$ and a pulsed white line laser (WLL, for $\lambda=488,561$ and $633 \mathrm{~nm}$ ). The cells were seeded on poly-L-lysine coated glass coverslips $(12 \times 12 \mathrm{~mm})$ placed inside 6-well plates $\left(3 \mathrm{~mL}, 5 \times 10^{4}\right.$ cells/well $)$ and grown for $24 \mathrm{~h}$ at standard culture conditions. Afterwards, the MPA-capped CdTeSe QDs at the desired concentration were internalized $\left(200 \mu \mathrm{L}, 10^{-9} \mathrm{M}\right)$, and the incubation protocol was conducted as described for the cytocompatibility experiments. At $24 \mathrm{~h}$ of incubation, the MPA-CdTeSe QDs-containing cells were washed and stained for visualization with DAPI (cell nucleus) and Bodipy Phalloidin (cytoplasm). Briefly, $350 \mathrm{~mL}$ of paraformaldehyde ( $\% w / v$ in $1 \times$ PBS) were added to each coverslip, incubated for $10 \mathrm{~min}$, and washed with $1 \times$ PBS. Then, $350 \mathrm{~mL}$ of a Triton X-100 solution $(0.1 \% w / v$ in $1 \times$ PBS) were added, incubated for $10 \mathrm{~min}$, and washed with $1 \times$ PBS. Then, $200 \mu \mathrm{L}$ of Bodipy Phalloidin 650/665 (5\% $v / v$ in $1 \times$ PBS) were added, incubated for $20 \mathrm{~min}$ in darkness, and washed 
with $1 \times$ PBS. Then, the attached cells were exposed to one drop of ProLong Gold antifade loaded with DAPI and cured for $24 \mathrm{~h}$ at $-20^{\circ} \mathrm{C}$ in darkness. Excitation lines of 405 (emissions at $425-475 \mathrm{~nm}$ ) and $633 \mathrm{~nm}$ (emissions at 650-725 nm) were employed for the visualization of cellular nucleus (stained with 4',6-diamidino-2-phenylindole (DAPI), from Invitrogen, Carlsbad, CA, USA) and cytoplasm (stained with Bodipy Phalloidin from Invitrogen, Carlsbad, NM, USA), respectively. All experiments were carried out in duplicate.

\section{Results and Discussion}

\subsection{Synthesis of Near-Infrared MPA-Capped CdTe and CdTeSe QDs}

Two important challenges in the synthesis of QDs for their application in biological systems have been to create QDs that (1) are biocompatible and (2) emit in the near infrared. However, many of the synthetic methods reported using organic solvents, which involves a second step for the modification of the QD surface with thiolated ligands to make it water soluble. Although thiol-based ligands have proven stable surface ligands for long-term water solubility, they fail to conserve the high QYs of QDs [34,35]. There is always a significant reduction in the luminescence efficiency following the transition from organic solvents into water (QY is usually below 20\%) [36]. For this reason, the direct aqueous synthesis of MPA-capped QDs using a single-step and ambient atmosphere methodology was performed. The synthesis conditions were modified with the objective to optimize the QY of the QDs. Then, QDs of CdTeSe were obtained at different molar ratios of Te:Se (1:0, 0.75:0.25, and 0.50:0.50) and different reaction times $(15,30,60$, and $90 \mathrm{~min})$. Figure S1 shows images of the as-prepared QDs under ambient light and UV light. A clear red-shift in the emission wavelength is seen for longer reaction times, indicating that larger QDs have formed.

\section{Characterization by UV-VIS and Fluorescence Spectroscopy}

Absorption and fluorescence spectra of the MPA-capped QDs re-dispersed in water at $\mathrm{pH} 8$ were obtained. As can be appreciated in Figure 1, when the reaction time increases and the Te proportion is lower, the emission wavelengths shift to the near-infrared zone. Unlike binary QDs, the growth kinetics of alloyed CdTeSe QDs are dependent upon three chemical reactants (Cd, Te, and Se) and their relative concentrations in the reaction [12]. It has been proven that elemental Te is considerably more reactive than Se toward $\mathrm{Cd}$ under rapid nucleation and that equilibrium between Te and Se are reached at higher reaction times [10]. The change in the Te and Se composition during QD growth was reflected on the QDs emission wavelengths.
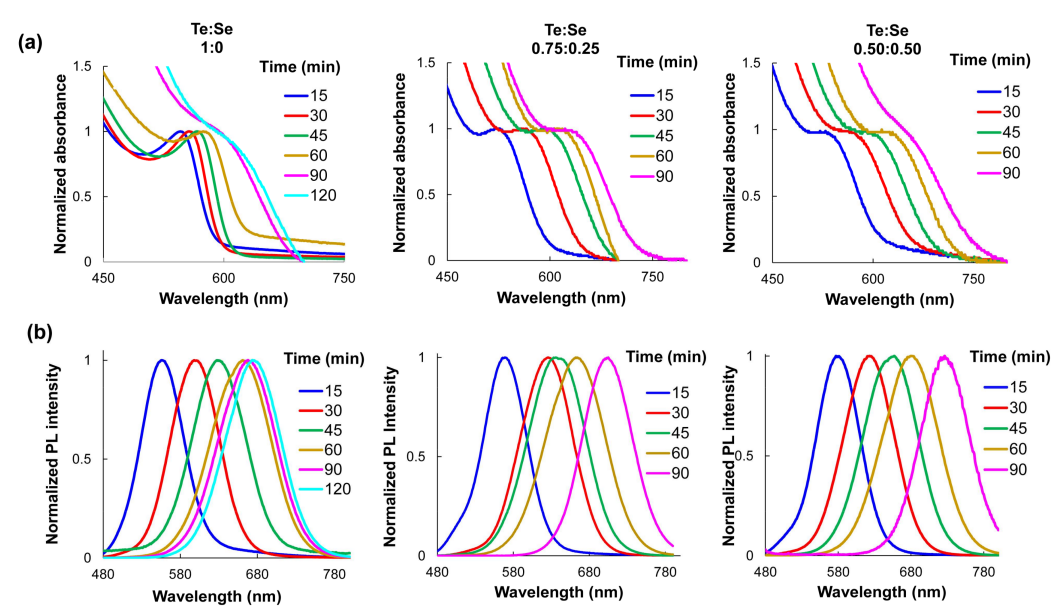

Figure 1. Evolution of (a) absorption and (b) fluorescence spectra of mercaptopropionic acid (MPA)-capped CdTeSe quantum dots (QDs) in water at $\mathrm{pH} 8$ obtained at different molar ratios of Te:Se $(1: 0,0.75: 0.25,0.50: 0.50)$ and different time reactions. 
The mechanism of the QDs' formation can be described as follows; during the first step MPA coordinates with $\mathrm{Cd}^{2+}$ ions to form the Cd-MPA complex and to prevent the deposition of non-soluble $\mathrm{Cd}$ compounds. The addition of $\mathrm{NaBH}_{4}$ results in the reduction of $\mathrm{TeO}_{3}{ }^{2-}$ to $\mathrm{Te}^{2-}$ and the reduction of elemental Se to $\mathrm{Se}^{2-}$. The precursor of $\mathrm{Te}\left(\mathrm{Te}^{2-}\right)$ rapidly reacts (twice as fast as $\mathrm{Se}^{2-}$ ) resulting CdTe-rich nuclei QDs initially. As the reaction progresses, CdSe is formed on the core, and an alloyed structure is produced. MPA ligands tightly bind to the QDs' surfaces.

Similar to the change in the emission wavelength, QYs become larger as the reaction time increases from 15 to $60 \mathrm{~min}$ and the Te:Se molar ratio decreases (Table S1). The highest QY, 84\%, was obtained with a molar relation of 0.75:0.25 of Se:Te and a reaction time of $60 \mathrm{~min}$. These are remarkable results since few investigations have obtained comparable QY values for water-soluble QDs [37]. The MPA-capped QDs' diameters were estimated using the absorption peaks and the empirical equation previously reported by Peng [32] at different reaction times (see Table S1). STEM images of the prepared MPA-capped CdTe and CdTeSe QDs (Figure 2) indicate uniformly dispersed, spherical QDs. The results obtained from the analysis of the size distribution histograms of the QDs show that the average crystal size was $3.4 \pm 0.23,3.8 \pm 0.25$, and $4.1 \pm 0.2 \mathrm{~nm}$, confirming the results obtained by the Peng regression.

Nanoparticle composition was further examined by EDS analysis. The presence of $\mathrm{Cd}$, $\mathrm{Te}$, and $\mathrm{Se}$ was confirmed by the presence of characteristic peaks of these elements (Figure S2). The remaining signals correspond to lattice elements of the sample support.
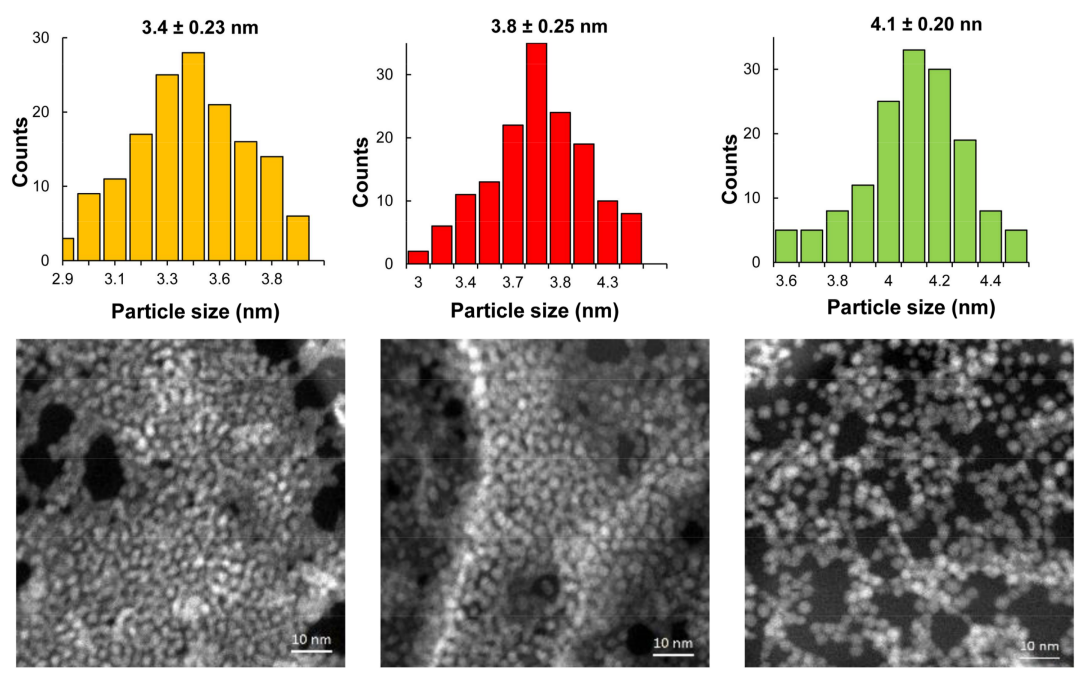

Figure 2. Scanning transmission electron microscopy (STEM) images obtained by Z contrast of the MPA-capped QDs at different molar ratios of Te:Se (1:0, 0.75:0.25, 0.50:0.50) and time reaction of $60 \mathrm{~min}$. Above: the corresponding histogram of size distribution.

Moreover, the obtained QDs dispersed in the phosphate buffer $(\mathrm{pH} 8)$ showed high optical stability when irradiated under xenon ion laser at $365 \mathrm{~nm}$ UV light for $60 \mathrm{~min}$. Figure 3 shows the dependence of the relative fluorescence intensity of the MPA-capped CdTe and MPA-capped CdTeSe QDs with the UV irradiation time. Previous studies have shown that unsaturated Te and Se atoms on QDs' surfaces may be oxidized under UV light exposure, which may lead to possible quenching of QDs' fluorescence [38]. The as-prepared QDs (binary and ternary) showed no decrease in fluorescence intensity. In fact, a photo enhancement of $15 \%$ was observed for the MPA-capped CdTe QDs in the first 10 minutes, while the fluorescence of the alloyed MPA-capped CdTeSe remained practically constant throughout the irradiation time interval. The differences in luminescence can therefore be attributed to different surface properties [6]. The alloyed MPA-capped CdTeSe QDs with gradient distribution of components consisting of Te-rich inner cores and Se-rich outer shells contain fewer trap sites or quenching defects on the surface. 
The photo brightening of the MPA-capped QDs after UV irradiation has been associated with the photo-adsorption of water molecules onto nanocrystals' surfaces providing restoring functions on the QDs' surfaces, eliminating photoluminescence (PL)-quenching defects or trap sites [39]. These results confirm that there are fewer trap sites or quenching defects on the surfaces of ternary QDs. Based on this behavior, the MPA-capped CdTeSe QDs were selected for further FRET studies.

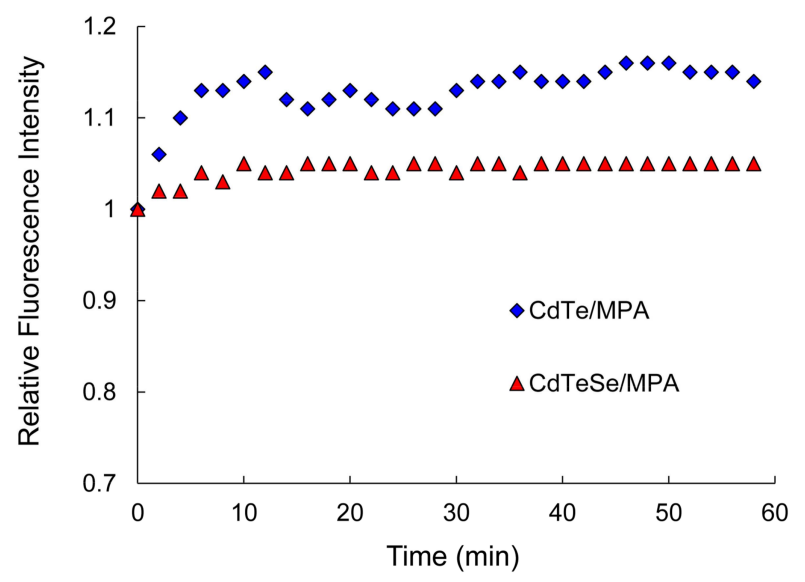

Figure 3. Photostability of the MPA-capped CdTe and the MPA-capped CdTeSe QDs in water at $\mathrm{pH} 8$ under UV light irradiation.

\subsection{Förster Resonance Energy Transfer FRET Studies}

The potential of QDs as fluorescent energy donors in FRET assays was explored by designing four systems of MPA-capped CdTeSe QDs (QDs626, QDs629, QDs636, and QDs663) as energy donors and Cy5 dye as energy acceptors. According to the Förster resonant energy transfer theory, a condition that affects the efficiency of energy transfer is the overlap between the donor emission spectrum and the acceptor absorption spectrum. The absorption spectrum of the Cy5 dye and emission spectra of the four MPA-capped CdTeSe QDs (spectra are normalized) are shown in Figure 4. As can be seen, the emission spectra of the MPA-capped CdTeSe QDs and the absorption spectrum of the Cy5 showed a large spectral overlap.

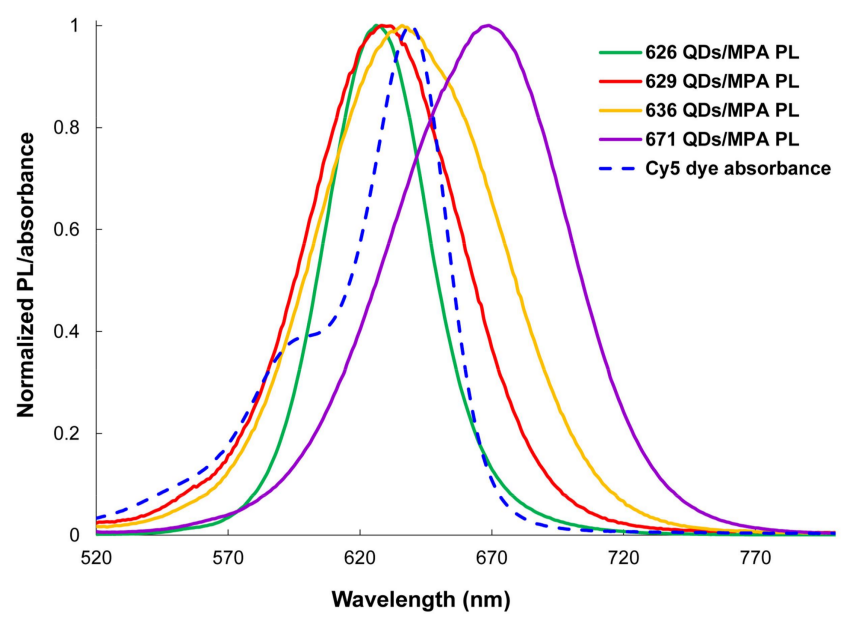

Figure 4. Spectral overlap of the donor MPA-capped CdTeSe QDs emissions and the acceptor Cy5 dye absorption. The solid lines represent the emission spectra of the donors $\left(\lambda_{\mathrm{em}}=626,629,636\right.$, and $671 \mathrm{~nm})$ and the dotted line represents the absorption spectrum of the Cy5 dye $\left(\lambda_{\mathrm{em}}=639 \mathrm{~nm}\right)$. 


\subsection{Selection of the Optimal Conditions for the FRET Process}

The optimal wavelength to excite the FRET pair is the one for which only the donor is excited, and the acceptor excitation is minimal. As can be observed in the absorption spectrum of the dye in the Figure 5a, this wavelength is $450 \mathrm{~nm}$. In Figure 5b, a three dimensional (3D) spectrum produced for the MPA-capped CdTeSe and the dye solution with a molar ratio of 1:20, respectively, is shown. The 3D spectra in Figure 5b show that the optimum excitation wavelength of the QDs is at $450 \mathrm{~nm}$, while the optimum excitation wavelength of the dye is $630 \mathrm{~nm}$.

(a)

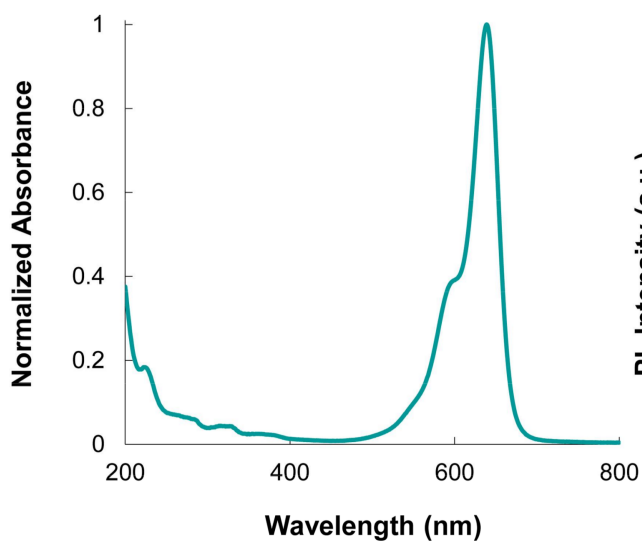

(b)

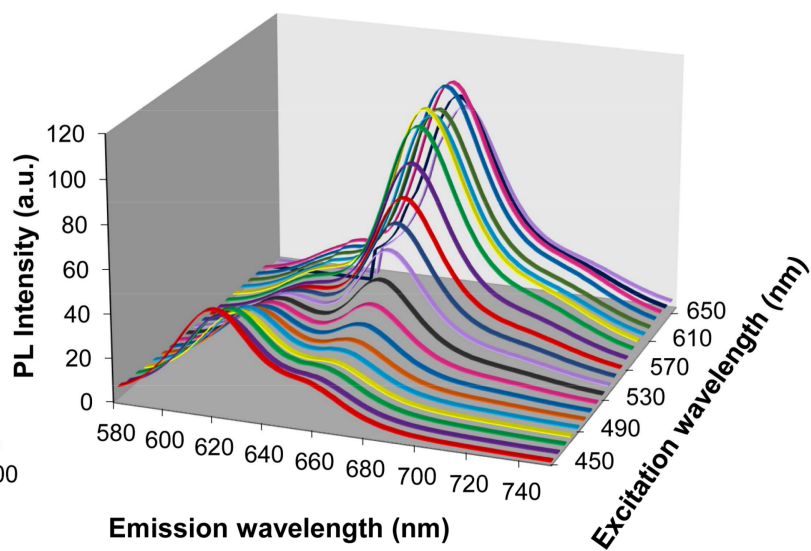

Figure 5. (a) Absorption spectrum of the Cy5 dye and (b) the MPA-capped CdTeSe-Cy5 conjugate 3D spectra.

\subsection{Zeta Potential}

With the objective to corroborate the surface charge on the QDs and the electrostatic interactions between the MPA-capped CdTeSe QDs and the Cy5 dye, Z potential was determined by varying the $\mathrm{pH}$ of the QDs (Figure 6a) and adding successive amounts of the Cy5 dye (Figure 6b).

(a)

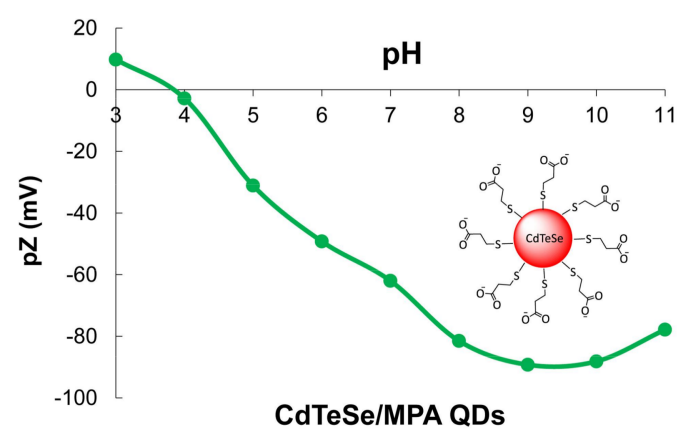

(b)

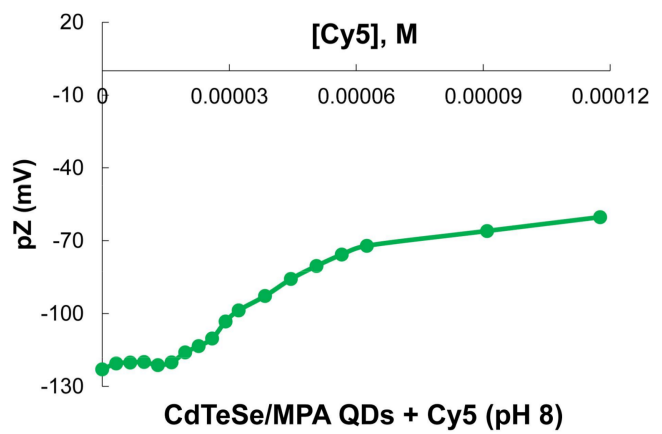

Figure 6. (a) Potential Z of the MPA-CdTeSe QDs at different $\mathrm{pH}$ values and (b) the titration of the MPA-CdTeSe QDs with the Cy5 at $\mathrm{pH} 8$.

The MPA-capped QDs have negative charge between $\mathrm{pH} 4-11$ (Figure 6a), due to carboxylate groups on their surfaces. An isoelectric point is localized at $\mathrm{pH} 4$ coinciding with the $\mathrm{pKa}$ of the carboxylic acid. The successive additions of the dye to the MPA-QDs, lead to a decrease in the QDs' negative surface charge $(-60 \mathrm{mv})$ upon addition of increasing amounts of the Cy5 dye, indicating complex formation (Figure 6b). This behavior confirms the electrostatic interactions between the positively-charged dye and the negatively-charged nanoparticles, decreasing the total charge of the 
MPA-QDs. The MPA-capped CdTeSe QDs bond with the Cy5 dye via electrostatic interactions between their carboxylate groups and the iminium group of $\mathrm{Cy} 5$ dye. In the phosphate buffer ( $\mathrm{pH} 8$ ), stable conjugates are formed.

\subsection{MPA-Capped CdTeSe QDs' Titrations of Different Sizes with Cy5 Dye}

The influence of the QDs' sizes on the FRET process was studied by successive titrations of different sizes of QDs (QDs626, QDs629, QDs636, and QDs671) with the Cy5 dye. A decrease in the QDs' emissions and the enhancement of the Cy5 dye emission was only seen for QDs629 and QDs636, indicating a local interaction between the QDs and the Cy5 dye, as well as a FRET process for these conjugates. For the conjugates between QDs626 and QDs663, = the typical behavior of a FRET process was not shown. Figure 7 shows the emission spectra of the successive titration of the MPA-capped CdTeSe QDs (629 and 636) with the Cy5 dye $\left(\lambda_{\mathrm{ex}}=450 \mathrm{~nm}\right)$.
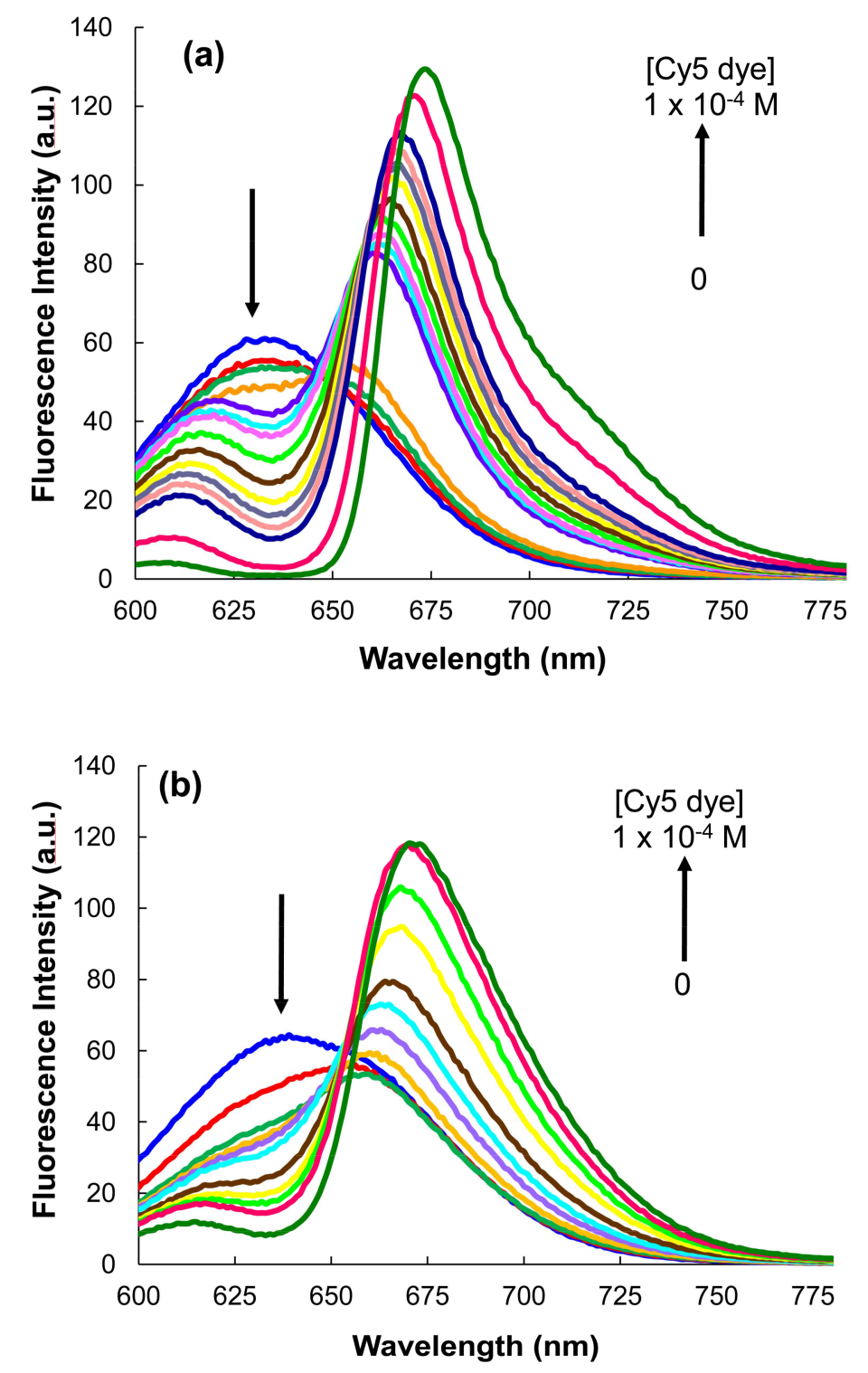

Figure 7. Titration emission spectra of the MPA-capped CdTeSe QDs with the Cy5 dye. (a) QDs629 and (b) QDs636.

Figure S3 illustrates the PL signals for the two sets of 629- and 636-nm-emitting QDs, as a function of the Cy5/QD molar ratio. The plots agree well with the experimental observations for the progressive 
donor PL quenching compared to the acceptor PL enhancement with increasing amounts of the Cy5 dye. Graphic analysis also shows that the enhancement of the fluorescence intensity of the Cy5 dye begins to show signs of saturation at a molar ratio of 50:1. The results coincide with previous studies, which have reported saturation molar ratios larger than 10:1 to 50:1 [40,41].

The energy transfer process between the QDs and the Cy5 dye was confirmed for the QD629-Cy5 and QD636-Cy5 FRET pairs. The fact that $R_{0}$ (the Förster distance) and $r$ (the distance between the QDs and the dye) values are in the range of $2-8 \mathrm{~nm}$ and that the required condition, $0.5 R_{0}<r<1.5 R_{0}$, was fulfilled, indicate the existence of the energy transfer from the QDs to the Cy5 dye in these cases [42]. For the QD626-dye and QD671-dye FRET pairs, the condition is not fulfilled. The MPA-capped QD629-Cy5 and MPA-capped QD636-Cy5 donor-acceptor pairs had good results with high efficiency, overlap area, and binding constant (Table 1). Based on these results, the QDs629 and QDs636 can be used in FRET assays with the Cy5 dye in biological applications.

Table 1. Calculated overlap integrals $(J)$, FRET efficiencies $(E)$, Förster distances $\left(R_{0}\right)$, distance between the donor and the acceptor $(r)$, and binding constant $(K)$ obtained by Equations (S1)-(S4) (Supplementary Materials).

\begin{tabular}{|c|c|c|c|c|c|c|c|}
\hline MPA-QDs & $\lambda_{\mathrm{em}}(\mathrm{nm})$ & $\mathrm{D}_{\text {UV }}(\mathrm{nm})$ & $J\left(\mathrm{~cm}^{3} \mathrm{Lmol}^{-1}\right)$ & $E$ & $R_{0}(\mathrm{~nm})$ & $r(\mathrm{~nm})$ & $K$ \\
\hline QD629 & 629 & 3.48 & $2.01 \times 10^{-12}$ & 0.96 & 4.37 & 2.58 & $2.30 \times 10^{4}$ \\
\hline QD636 & 636 & 3.63 & $1.88 \times 10^{-12}$ & 0.88 & 4.41 & 3.14 & $8.24 \times 10^{4}$ \\
\hline
\end{tabular}

\subsection{Citocompatibility of the CdTeSe/MPA QDs}

Figure 8 shows the viability of HeLa cells after exposure to the MPA-capped CdTe and MPA-capped CdTeSe QDs as a function of particle concentration. This figure reflects that both QDs display a dose dependent cytocompatibility typical of nanomaterials, depicting a LC50 (lethal concentration for $50 \%$ of the cells) of about $10^{-8} \mathrm{M}$ and a MinTC (minimum toxic concentration) of ca. $10^{-9} \mathrm{M}$, below which cell growth inhibition appears to be negligible. These results are in good agreement with those presented by $\mathrm{Hu}$ et al. [43], who did not observe cytotoxicity for four CdSe/ZnS QDs coated with PEG within the concentration range of 10-100 $\mathrm{nM}$ upon internalization to human hepatocellular carcinoma (HepG2) cells [42]. The authors attributed this outstanding outcome to the structure of the $\mathrm{ZnS}$ shell and the PEG coating, which are expected to avoid the release of Cd from the QDs' cores. In our case, such a biocompatible coating is rendered by the MPA, which also endowed the QDs with a zeta-potential of about $-65 \mathrm{mV}$ (see Figure 6), protecting the cells from the membrane disruption phenomenon commonly observed upon their interaction with positively-charged compounds. In our results, better cytocompatibility is found for the ternary QDs than for the binary QDs, which is in agreement with the higher stability of the ternary QDs (Figure 3).

Assessing the QDs' potential toxicity is not a simple matter. A number of publications agree that the main toxicity sources in $\mathrm{Cd}^{2+}$-containing QDs are $\mathrm{Cd}^{2+}$ ion release and reactive oxygen species (ROS) production. Kauffer et al. [44] compared the ability of ternary alloyed QDs (mercaptosuccinic acid (MSA)-capped CdSeS QDs) versus binary QDs (MSA-CdSe) to generate light-induced ROS. The binary QDs produced $\cdot \mathrm{OH}$ radicals immediately, whereas the ternary QDs required extensive irradiation times and presented less photobleaching. The third element in alloyed QDs plays a major role in providing a higher structural and chemical stability; this may result not only in less ROS production but also in a decrease of $\mathrm{Cd}^{2+}$ release. 


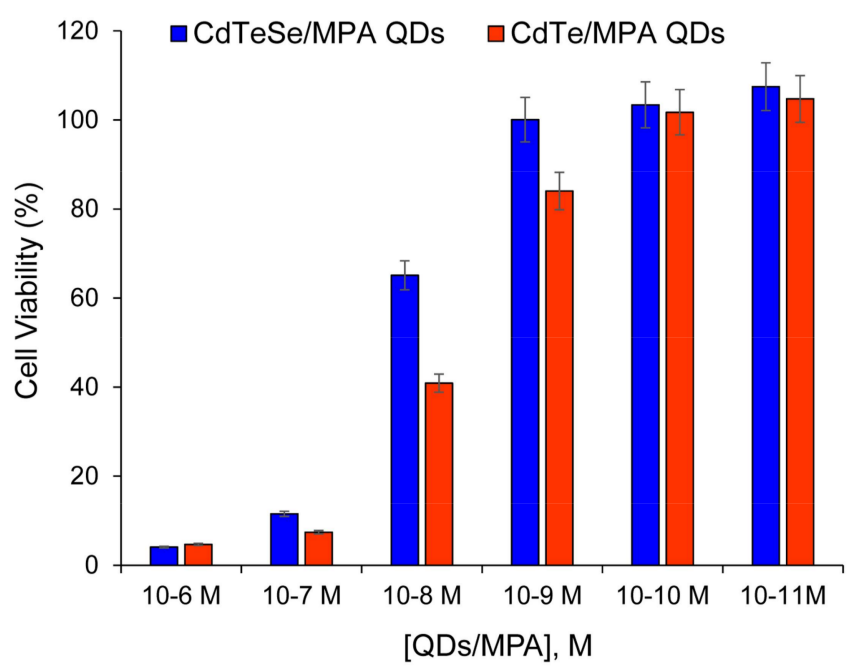

Figure 8. Viability of human cancer cells (HeLa) cells after exposure to the MPA-capped CdTe and CdTeSe QDs.

\subsection{Cell Internalization of the MPA-Capped CdTeSe QDs}

Figure 9 shows the internalization of the MPA-capped CdTeSe QDs at $6 \mathrm{~h}$ post-internalization at a concentration of $10^{-9} \mathrm{M}$. This figure reflects two features that are worthy of discussion. Firstly, the displayed image shows a high population of cells presenting a healthy appearance in presence of the QDs after internalization, even during mitosis when they are known to be especially sensitive to any external stimuli [45]. This finding confirms the CCK-8 results identifying the MPA-capped CdTeSe QDs as cytocompatible. Second, the QDs homogeneously accumulated all over the cytoplasm (depicted as black dots in reflection mode), demonstrating these ternary materials as suitable and cytocompatible candidates for cell staining.

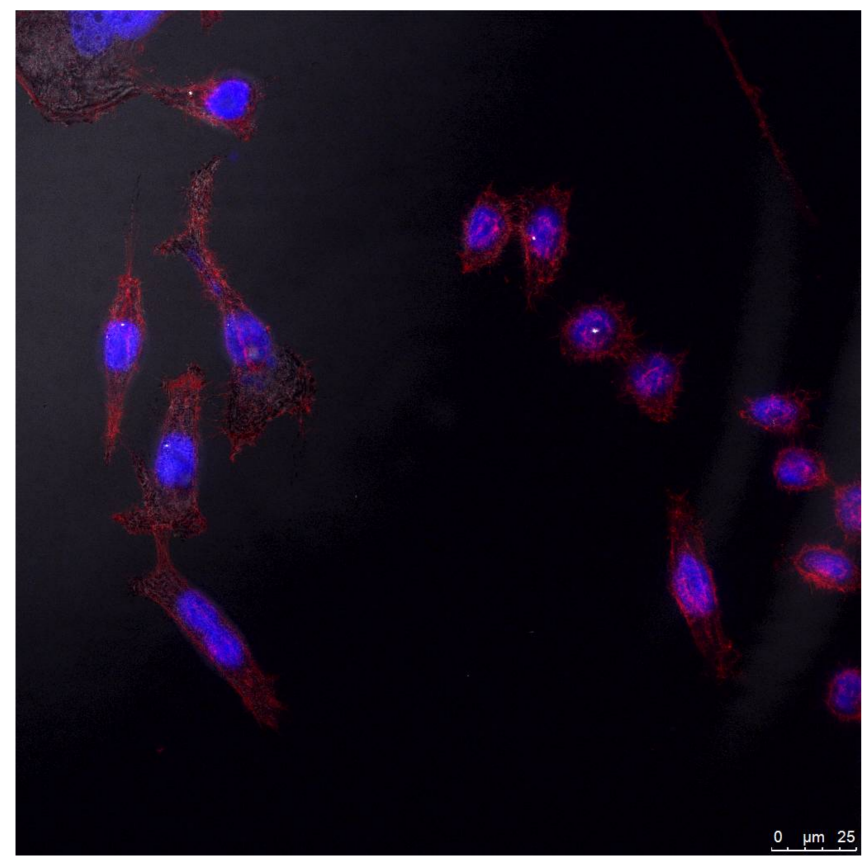

Figure 9. Confocal microscopy image in reflection mode of HeLa cells upon exposure to the MPA-capped CdTeSe QDs. Stained in blue and red are the cell nucleus and cytoplasm, respectively. QDs are observed in reflection mode as black dots all over the cytoplasm. 


\section{Conclusions}

We have synthesized high quantum yield and stable NIR-emitting alloyed MPA-capped CdTeSe QDs that are water soluble using a facile and direct modified synthetic route. PL properties of the QDs were influenced by reaction time and Te:Se composition. The MPA-capped CdTeSe QDs exhibited a red-shifted emission, higher QYs, and better stability and cytocompatibility than corresponding MPA-capped CdTe QDs. By careful selection, different sized MPA-capped CdTeSe QDs (energy donors) and Cy5 dye (energy acceptor) were used for FRET assays. All factors for effective FRET were studied, such as QD size, proximity between the donor and the acceptor pair (electrostatic interactions), and high spectral overlap. The occurrence of FRET was confirmed in the conjugates of MPA-capped CdTeSe 629 nm QDs with Cy5 and in MPA-capped CdTeSe 636 nm QDs with Cy5 with good efficiency.

Very importantly, as depicted by the CCK-8 assay and confocal microscopy, the QDs were demonstrated to be highly cytocompatible and capable of ordering homogeneously and in high proportions all over the cytoplasm. The outstanding cytocompatibility of the QDs studied, the good efficiency of the FRET process, and their stability make the proposed system a good candidate for the development of FRET-based analytical tools such as fluoroimmunoassays.

Supplementary Materials: The following are available online at http://www.mdpi.com/2079-4991/8/4/231/s1. Figure S1: Images of the QDs under ambient light and UV light. Figure S2: EDS spectra of the CdTeSe QDs at different molar ratios of Te:Se (1:0, 0.75:0.25, 0.50:0.50) and time reaction of $60 \mathrm{~min}$. Figure S3: Experimental values for the donor relative PL intensity decay (red line) and acceptor PL intensity gain (blue line) versus molar ratio [Cy5]/[QD]. Table S1: Optical characterization of the CdTeSe QDs. FRET calculations and Equations (S1)-(S4).

Acknowledgments: G.P.-L. gratefully acknowledge support from Consejo Nacional de Ciencia y Tecnología (CONACyT), México (Project 167139), Tecnológico Nacional de México (TecNM) (Project 6281.17-P), and Supramolecular Chemistry Network (CONACyT, Project 271884). E.R.-V. thanks PRODEP (UABC) through Project NPTC 2017-305/488/E. M.A.-M. thanks funding from CONACyT (Mexico) through Research Projects INFR-2015-251863 and PDCPN-2015-89. P.T. thanks AEI and FEDER for research project MAT2016-80266-R and additional funding. D. Ramírez-Herrera thanks CONACyT for a doctoral fellowship.

Author Contributions: G.P.-L. conceived and supervised the project; D.E.R.-H. and E.R.-V. performed the experiments; F.P.-D. performed the TEM and EDS analysis; G.P.-L, D.E.R.-H., M.A.-M., F.P.-D., A.T.-G., P.T., and E.R.-V. analyzed the data and discussed the results; G.P.-L., D.E.R.-H., and M.A.-M. wrote the manuscript with feedback from all authors; P.T. corrected and improved the manuscript; G.P.-L., F.P.-D., and P.T. contributed reagents/materials/analysis tools.

Conflicts of Interest: The authors declare no conflict of interest.

\section{References}

1. Smith, A.M.; Nie, S.M. Semiconductor Nanocrystals: Structure, Properties, and Band Gap Engineering. Acc. Chem. Res. 2010, 43, 190-200. [CrossRef] [PubMed]

2. Xing, Y.; Rao, J. Quantum Dot Bioconjugates for in vitro Diagnostics \& in vivo Imaging. Cancer Biomark. 2008, 4, 307-319. [PubMed]

3. Medintz, I.L.; Mattoussi, H.; Clapp, A.R. Potential Clinical Applications of Quantum Dots. Int. J. Nanomed. 2008, 3, 151-167.

4. Michalet, X.; Pinaud, F.; Bentolila, L.; Tsay, J.; Doose, S.; Li, J.; Sundaresan, G.; Wu, A.M.; Gambhir, S.S.; Weiss, S. Quantum Dots for Live Cells, in vivo Imaging, and Diagnostics. Science 2005, 307, 538-544. [CrossRef] [PubMed]

5. Robel, I.; Subramanian, V.; Kuno, M.; Kamat, P.V. Quantum Dot Solar Cells. Harvesting Light Energy with CdSe Nanocrystals Molecularly Linked to Mesoscopic TiO 2 Films. J. Am. Chem. Soc. 2006, 128, 2385-2393. [CrossRef] [PubMed]

6. McDonald, S.A.; Konstantatos, G.; Zhang, S.; Cyr, P.W.; Klem, E.J.D.; Levina, L.; Sargent, E.H. Solution-processed PbS Quantum Dot Infrared Photodetectors and Photovoltaics. Nat. Mater. 2005, 4, 138-142. [CrossRef] [PubMed]

7. Frangioni, J.V. In vivo near-infrared Fluorescence Imaging. Curr. Opin. Chem. Biol. 2003, 7, 626-634. [CrossRef] [PubMed] 
8. Yang, F.; Yang, P.; Zhang, L. Synthesis of Near-Infrared-Emitting CdTeSe and CdZnTeSe Quantum Dots. Luminescence 2012, 28, 836-841. [CrossRef] [PubMed]

9. Cassette, E.; Helle, M.; Bezdetnaya, L.; Marchal, F.; Dubertret, B.; Pons, T. Design of New Quantum Dot Materials for Deep Tissue Infrared Imaging. Adv. Drug Deliv. Rev. 2013, 65, 719-731. [CrossRef] [PubMed]

10. Bailey, R.E.; Nie, S. Alloyed Semiconductor Quantum Dots: Tuning the Optical Properties without Changing the Particle Size. J. Am. Chem. Soc. 2003, 125, 7100-7106. [CrossRef] [PubMed]

11. Bailey, R.E.; Strausburg, J.B.; Nie, S. A New Class of Far-Red and Near-Infrared Biological Labels Based on Alloyed Semiconductor Quantum Dots. J. Nanosci. Nanotechnol. 2004, 4, 569-574. [CrossRef] [PubMed]

12. Jiang, W.; Singhal, A.; Wang, C.; Chan, W.C.W. Optimizing the Synthesis of Red- to Near-IR-Emitting CdS-Capped CdTe $\mathrm{Se}_{1-\mathrm{x}}$ Alloyed Quantum Dots for Biomedical Imaging. Chem. Mater. 2006, 18, 4845-4854. [CrossRef]

13. Pons, T.; Lequeux, N.; Mahler, B.; Sasnouski, S.; Fragola, A.; Dubertret, B. Synthesis of Near-Infrared-Emitting, Water-Soluble CdTeSe/CdZnS Core/Shell Quantum Dots. Chem. Mater. 2009, 21, 1418-1424. [CrossRef]

14. Wang, R.; Calvignanello, O.; Ratcliffe, C.I.; Wu, X.; Leek, D.M.; Zaman, M.B.; Kingston, D.; Ripmeester, J.A.; $\mathrm{Yu}, \mathrm{K}$. Homogeneously-Alloyed CdTeSe Single-Sized Nanocrystals with Bandgap Photoluminescence. J. Phys. Chem. C 2009, 113, 3402-3408. [CrossRef]

15. Talapin, D.V.; Mekis, I.; Gotzinger, S.; Kornowski, A.; Benson, O.; Weller, H. CdSe/CdS/ZnS and CdSe/ZnSe/ZnS Core-Shell-Shell Nanocrystals. J. Phys. Chem. B 2004, 108, 18826-18831. [CrossRef]

16. Sperling, R.A.; Parak, W.J. Surface Modification, Functionalization and Bioconjugation of Colloidal Inorganic Nanoparticles. Philos. Trans. R. Soc. A 2010, 368, 1333-1383. [CrossRef] [PubMed]

17. Love, J.C.; Estroff, L.A.; Kriebel, J.K.; Nuzzo, R.G.; Whitesides, G.M. Self-Assembled Monolayers of Thiolates on Metals as a Form of Nanotechnology. Chem. Rev. 2005, 105, 1103-1169. [CrossRef] [PubMed]

18. Ma, K.G.; Bai, J.Y.; Fang, T.; Guo, H.Q. Synthesis of High-Quality Water-Soluble Near-Infrared-Emitting CdTe Quantum Dots Capped with 3-Mercaptobutyric Acid. J. Nanosci. Nanotechnol. 2014, 14, 4940-4948. [CrossRef] [PubMed]

19. Ma, K.G.; Fang, T.; Bai, J.Y.; Guo, H.Q. Regulating Properties of Quantum Dots: Effect of Methyl Side Groups of Mercapto Acids. RSC Adv. 2013, 3, 4935-4939. [CrossRef]

20. Liu, S.; Zhang, H.; Qiao, Y.; Su, X. One-Pot Synthesis of Ternary CuInS 2 Quantum Dots with Near-Infrared Fluorescence in Aqueous Solution. RSC Adv. 2012, 2, 819-825. [CrossRef]

21. Su, Y.; He, Y.; Lu, H.; Sai, L.; Li, Q.; Li, W.; Wang, L.; Shen, P.; Huang, Q.; Fan, C. The Cytotoxicity of Cadmium Based, Aqueous Phase-Synthesized, Quantum Dots and its Modulation by Surface Coating. Biomaterials 2009, 30, 19-25. [CrossRef] [PubMed]

22. Pan, Z.; Zhao, K.; Wang, J.; Zhang, H.; Feng, Y.; Zhong, X. Near Infrared Absorption of CdSe $\mathrm{Te}_{1-\mathrm{x}} \mathrm{Alloyed}$ Quantum Dot Sensitized Solar Cells with More than 6\% Efficiency and High Stability. ACS Nano 2013, 7, 5215-5222. [CrossRef] [PubMed]

23. Peynshaert, K.; Soenen, S.J.; Manshian, B.B.; Doak, S.H.; Braeckmans, K.; de Smedt, S.C.; Remaut, K. Coating of Quantum Dots Strongly Defines their Effect on Lysosomal Health and Autophagy. Acta Biomater. 2017, 48, 195-205. [CrossRef] [PubMed]

24. Su, Y.; Hub, M.; Fan, C.; He, Y.; Li, Q.; Li, W.; Wang, L.-H.; Shen, P.; Huang, Q. The Cytotoxicity of CdTe Quantum Dots and the Relative Contributions from Released Cadmium Ions and Nanoparticle Properties. Biomaterials 2010, 31, 4829-4834. [CrossRef] [PubMed]

25. Liu, J.; Hu, R.; Liu, J.; Zhang, B.; Wang, Y.; Liu, X.; Law, W.-C.; Liu, L.; Ye, L.; Yong, K.-T. Cytotoxicity Assessment of Functionalized CdSe, CdTe and InP Quantum Dots in Two Human Cancer Cell Models. Mater. Sci. Eng. C 2015, 57, 222-231. [CrossRef] [PubMed]

26. Chen, N.; He, Y.; Su, Y.; Li, X.; Huang, Q.; Wang, H.; Zhang, X.; Tai, R.; Fan, C. The Cytotoxicity of Cadmium-Based Quantum Dots. Biomaterials 2012, 33, 1238-1244. [CrossRef] [PubMed]

27. Du, J.; Li, X.; Wang, S.; Wu, Y.; Hao, X.; Xu, C.; Zhao, X. Microwave-Assisted Synthesis of Highly Luminescent Glutathione-Capped $\mathrm{Zn}_{1-\mathrm{x}} \mathrm{Cd}_{\mathrm{x}} \mathrm{Te}$ Alloyed Quantum Dots with Excellent Biocompatibility. J. Mater. Chem. 2012, 22, 11390-11395. [CrossRef]

28. Xue, B.; Deng, D.-W.; Cao, J.; Liu, F.; Li, X.; Akers, W.; Achilefu, S.; Gu, Y.-Q. Synthesis of NAC Capped Near Infrared-Emitting CdTeS Alloyed Quantum Dots and Application for in vivo Early Tumor Imaging. Dalton Trans. 2012, 41, 4935-4947. [CrossRef] [PubMed] 
29. Medintz, I.L.; Clapp, A.R.; Mattoussi, H.; Goldman, E.R.; Fisher, B.; Mauro, J.M. Self-Assembled Nanoscale Biosensors Based on Quantum Dot FRET Donors. Nat. Mater. 2003, 2, 630-638. [CrossRef] [PubMed]

30. Sapsford, K.E.; Berti, L.; Medintz, I.L. Materials for Fluorescence Resonance Energy Transfer Analysis: Beyond Traditional Donor-Acceptor Combinations. Angew. Chem. Int. Ed. 2006, 45, 4562-4588. [CrossRef] [PubMed]

31. Oluwatobi, O.; Daramola, O.A.; Ncapayi, V. A Facile Green Synthesis of Type II Water Soluble CdTe/CdS Core Shell Nanoparticles. Mater. Lett. 2014, 133, 9-13.

32. Yu, W.W.; Qu, L.H.; Guo, W.Z.; Peng, X.G. Experimental determination of the extinction coefficient of CdTe, CdSe and CdS nanocrystals. Chem. Mater. 2003, 15, 2854-2860. [CrossRef]

33. Rodríguez-Velázquez, E.; Silva, M.; Taboada, P.; Mano, J.F.; Suárez-Quintanilla, D.; Alatorre-Meda, M. Enhanced Cell Affinity of Chitosan Membranes Mediated by Superficial Cross-Linking: A Straightforward Method Attainable by Standard Laboratory Procedures. Biomacromolecules 2014, 15, 291-301. [CrossRef] [PubMed]

34. Mattoussi, H.; Mauro, J.M.; Goldman, E.R.; Anderson, G.P.; Sundar, V.C.; Mikulec, F.V.; Bawendi, M.G. Self-Assembly of CdSe-ZnS Quantum Dot Bioconjugates Using an Engineered Recombinant Protein. J. Am. Chem. Soc. 2000, 122, 12142-12150. [CrossRef]

35. Pathak, S.; Choi, S.K.; Arnheim, N.; Thompson, M.E. Hydroxylated Quantum Dots as Luminescent Probes for in Situ Hybridization. J. Am. Chem. Soc. 2001, 123, 4103-4104. [CrossRef] [PubMed]

36. Gao, X.; Cui, Y.; Levenson, R.M.; Chung, L.W.K.; Nie, S. In vivo Cancer Targeting and Imaging with Semiconductor Quantum Dots. Nat. Biotechnol. 2004, 22, 969-976. [CrossRef] [PubMed]

37. Xiao, Q.; Huang, S.; Su, W.; Chan, W.H.; Liu, Y. Facile Synthesis and Characterization of Highly Fluorescent and Biocompatible $N$-acetyl-L-cysteine Capped CdTe/CdS/ZnS core/shell/shell Quantum Dots in Aqueous Phase. Nanotechnology 2012, 23, 495717. [CrossRef] [PubMed]

38. Borchert, H.; Talapin, D.V.; Gaponik, N.; McGinley, C.; Adam, S.; Lobo, A.; Moller, T.; Weller, H. Relations between the Photoluminescence Efficiency of CdTe Nanocrystals and Their Surface Properties Revealed by Synchrotron XPS. J. Phys. Chem. B 2003, 107, 9662-9668. [CrossRef]

39. Oda, M.; Tsukamoto, J.; Hasegawa, A.; Iwami, N.; Nishiura, K.; Hagiwara, I.; Ando, N.; Horiuchi, H.; Tani, T. Photobrightening of CdSe/ZnS/TOPO Nanocrystals. J. Lumin. 2007, 122-123, 762-765. [CrossRef]

40. Clapp, A.R.; Medintz, I.L.; Mauro, J.M.; Fisher, B.R.; Bawendi, M.G.; Mattoussi, H. Fluorescence Resonance Energy Transfer between Quantum Dot Donors and Dye-Labeled Protein Acceptors. J. Am. Chem. Soc. 2004, 126, 301-310. [CrossRef] [PubMed]

41. Chong, E.Z.; Matthews, D.R.; Summers, H.D.; Njoh, K.L.; Errington, R.J.; Smith, P.J. Development of FRET-Based Assays in the Far-Red Using CdTe Quantum Dots. J. Biomed. Biotechnol. 2007, 2007, 1-7. [CrossRef] [PubMed]

42. Lakowicz, J.R. Principles of Fluorescence Spectroscopy; Springer: New York, NY, USA, 2006; p. 678.

43. Peng, L.; He, M.; Chen, B.; Wu, Q.; Zhang, Z.; Pang, D.; Zhu, Y.; Hu, B. Cellular Uptake, Elimination and Toxicity of CdSe/ZnS Quantum Dots in HepG2 Cells. Biomaterials 2013, 34, 9545-9558. [CrossRef] [PubMed]

44. Kauffer, F.A.; Merlin, C.; Balan, L.; Schneider, R. Incidence of the Core Composition on the Stability, the ROS Production and the Toxicity of CdSe Quantum Dots. J. Hazard. Mater. 2014, 268, 246-255. [CrossRef] [PubMed]

45. Stobbe, C.C.; Park, S.J.; Chapman, J.D. The Radiation Hypersensitivity of Cells at Mitosis. Int. J. Rad. Biol. 2002, 78, 1149-1157. [CrossRef] [PubMed]

(C) 2018 by the authors. Licensee MDPI, Basel, Switzerland. This article is an open access article distributed under the terms and conditions of the Creative Commons Attribution (CC BY) license (http:/ / creativecommons.org/licenses/by/4.0/). 